\title{
An experimental study on providing a scientific evidence for seven-time alcohol- steaming of Rhei Rhizoma when clinically used
}

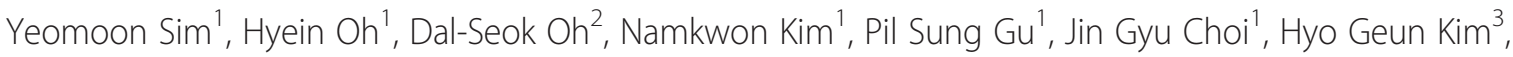
Tong Ho Kang ${ }^{4}$ and Myung Sook Oh ${ }^{1,3^{*}}$

\begin{abstract}
Background: Rhei Rhizoma (RR) has been widely used as laxative and processed to alter its therapeutic actions or reduce its side effects. In this study, we evaluated experimentally the clinical application guideline that RR should be alcohol-steamed seven times before being used in elderly patients, as described in Dongeuibogam, the most famous book on Korean traditional medicine.

Methods: Unprocessed RR (RR-U) was soaked in rice wine, steamed and then fully dried (RR-P1). The process was repeated four (RR-P4) or seven times (RR-P7). Reversed-phase high-performance liquid chromatography was used to determine the RR-U, RR-P1, RR-P4 and RR-P7 (RRs) constituents. To evaluate the effect of RRs on liver toxicity, human hepatoma cells (HepG2) were treated with RRs at $100 \mu \mathrm{g} / \mathrm{mL}$ for $4 \mathrm{~h}$ and then cell viabilities were measured using the 3-(4,5-dimethylthiazol-2-yl)-2,5-diphenyltetrazolium bromide method. To confirm the effects in vivo, 5-week-old male Sprague-Dawley rats were treated with RRs at $3 \mathrm{~g} / \mathrm{kg} /$ day for 21 days. Body weight and serum biochemical parameters were measured and liver histology was assessed.

Results: The levels of sennosides decreased in processed RRs in an iteration-dependent manner, while the emodin level was unaffected. In HepG2 cells, cell viability was reduced with RR-U, while the toxicity decreased according to the number of processing cycles. The changes in body weight, relative liver weight and liver enzymes of RR-U-treated rats were reduced in processed RRs-treated rats. Histopathological analysis indicated swelling and cholestasis improved following seven times alcohol-steaming cycles.
\end{abstract}

Conclusions: These results provide experimental evidence that RR-P7 almost completely reduces RR hepatotoxicity.

Keywords: Rhei Rhizoma, Alcohol-steaming, Hepatotoxicity, Dongeuibogam

\section{Background}

Rhei Rhizoma (RR) is a rhubarb rhizome and belongs to the genus Rheum in the family Polygonaceae. According to traditional literature, RR has a bitter taste and a cold property and has been commonly used to remove accumulation with purgation, clear heat and purge fire, cool

\footnotetext{
* Correspondence: msohok@khu.ac.kr

1Department of Life and Nanopharmaceutical Science, Graduate School, Kyung Hee University, 26 Kyungheedae-ro, Dongdaemun-gu, Seoul 02447 Republic of Korea

${ }^{3}$ Department of Oriental Pharmaceutical Science, College of Pharmacy and Kyung Hee East-west Pharmaceutical Research Institute, Kyung Hee University, 26 Kyungheedae-ro, Dongdaemun-gu, Seoul 02447, Republic of Korea

Full list of author information is available at the end of the article
}

the blood and remove toxins, expel stasis to unblock the meridian and drain dampness in Korea, China, Japan and other Asian countries [1]. RR contains dianthrone glycosides (sennosides A-F) and anthraquinones (chrysophanol, emodin, aloe-emodin, physcion and rhein) [2]. Several studies have investigated the pharmacological effects of RR, such as its purgative and cathartic, antiinflammatory, anti-oxidative and anti-microbial effects [3]. Although RR has been widely used for its various pharmacological actions, it is reported that RR at high doses is toxic to the liver and kidney $[4,5]$.

The processing of medicinal herbs is a common pharmaceutical technique that allows the use of different therapeutic modalities based on traditional theory; the 
most important purpose of herb processing is to enhance its efficacy and/or reduce toxicity [6]. Additionally, processing is important to moderate reactions (such as anaphylaxis and allergies), diminish adverse events and modify detrimental properties (such as disagreeable flavors and odors) [6]. Experimental studies have been conducted to confirm the benefits of processing medicinal herbs to reduce their toxicity $[7,8]$.

$\mathrm{RR}$ is frequently processed using various methods to alter its efficacy or reduce side effects before clinical application. Some recommended methods involve RR steaming after dipping or soaking in alcohol, stir-frying RR with alcohol or vinegar or carbonizing [9]. Wang et al. compared the levels of anthraquinones and tannins in several processed RR extracts using different methods and found RR steaming decreased combined anthraquinones, while there was little change in total anthraquinones and tannins. Additionally, water-steamed RR decreased the anthraquinone glycoside content $[7,8]$. Doui et al. reported that alcoholsoaking in $16 \%$ ethanol and then steaming of RR decreased the sennoside content, while the processing increased the anthraquinone content $[10,11]$. Most previous studies have focused on changes in the chemical constituents of processed RR.

Dongeuibogam, the most famous book on Korean traditional medicine, is an encyclopedic guideline of medical knowledge and treatment techniques first compiled by Joon Heo in 1613. As stated in this book, RR should be alcohol-steamed seven times when used for elderly patients [12]. In the present study, we evaluated the effects of seven-time alcohol-steaming on RR constituents and hepatotoxicity in rats and confirmed the statement in the book by providing experimental evidence. We measured the changes in constituents using reversed-phase highperformance liquid chromatography (RP-HPLC) and the effects on human hepatoma cell (HepG2) viability and body weight, blood chemistry and histopathological findings of rats subjected to various iterations of the $R R$ alcohol-steaming process.

\section{Methods}

\section{Materials}

Dulbecco's modified Eagle medium (DMEM), fetal bovine serum (FBS) and penicillin-streptomycin (P/S) were purchased from from Hyclone Laboratories, Inc. (Logan, UT, USA). Paraformaldehyde, 3-(4,5-dimethylthiazol-2-yl)-2,5diphenyltetrazolium bromide (MTT), and hematoxylin were purchased from Sigma-Aldrich (St. Louis, MO, USA). Other reagents and solvents were of guaranteed or analytical grade.

\section{Preparation of RR extract}

A dried rhizoma of Rheum palmatum was purchased from Kyung Hee Herb Pharm (Seoul, Korea); $500 \mathrm{~g}$ of unprocessed RR (RR-U) was soaked in $150 \mathrm{~g}$ of rice wine (14 \%; Lotte Liquor BG, Seoul, Korea) for $2 \mathrm{~h}$, steamed in a water bath for $2 \mathrm{~h}$, then fully dried in an oven at $40{ }^{\circ} \mathrm{C}$ (RR-P1). The process was repeated four (RR-P4) or seven times (RR-P7). Each sample was deposited in the herbarium of the College of Pharmacy at Kyung Hee University (DGM P0, P1, P4 and P7). RR-U, RR-P1, RR-P4 and RRP7 (RRs) were extracted using $70 \%$ ethanol for $24 \mathrm{~h}$ at room temperature. After leaching, the mixture was passed through filter paper (Whatman No. 2; Korea), concentrated under reduced pressure using a rotary vacuum evaporator, and then freeze-dried (FDU-550R; Eyela Co., Japan) to powder. The yields were 24.80, 28.70, 26.70 and $27.61 \%$, respectively. RRs were kept at $4{ }^{\circ} \mathrm{C}$. Before each experiment, the extracts were dissolved in an appropriate vehicle and were vortex-mixed for $2 \mathrm{~min}$ at room temperature.

\section{Analysis of RR constituents}

The sennoside A, sennoside B and emodin constituents in RRs were determined using RP-HPLC. The HPLC system consisted of a HPLC unit (LC-20A HPLC instrument, Shimadzu Co., Japan) and reversed-phase Shiseido CAPCELL PAK C18 UG120S column $(5 \mu \mathrm{m}, 4.6 \mathrm{~mm}$ I.D. $\times$ $25 \mathrm{~cm}$ ) was used for sennoside A detection and Biochoff chromatography column (ProntoSIL $250 \times 4.6 \mathrm{~mm}$ ) for sennoside B and emodin detection. For sennoside A detection, the mobile phase consisted of acetonitrile and $1.25 \%$ acetic acid at a flow rate of $0.6 \mathrm{~mL} / \mathrm{min}$, with elution of $80 \%$ acetic acid $\mathrm{v} / \mathrm{v}$ at a flow rate of $1 \mathrm{~mL} / \mathrm{min}$ at $30{ }^{\circ} \mathrm{C}$. For sennoside B detection, the mobile phase consisted of acetonitrile and $0.05 \mathrm{M}$ phosphoric acid at a flow rate of $1 \mathrm{~mL} / \mathrm{min}$, with gradient elution of $80 \%$ phosphoric acid v/v at a flow rate of $1 \mathrm{~mL} / \mathrm{min}$ at $40{ }^{\circ} \mathrm{C}$. For emodin detection, the mobile phase consisted of methanol and $1 \%$ phosphoric acid at a flow rate of $1 \mathrm{~mL} / \mathrm{min}$, with elution of $15 \%$ phosphoric acid v/v at a flow rate of $1 \mathrm{~mL} /$ min at $40{ }^{\circ} \mathrm{C}$. The injection volume was $10 \mu \mathrm{L}$.

\section{Effects of RRs on HepG2 cell viability}

HepG2 cells were obtained from the Korea Cell Line Bank (KCLB, Seoul, Korea). HepG2 cells were maintained in DMEM supplemented with $10 \% \mathrm{FBS}, 1 \% \mathrm{P} / \mathrm{S}$ in 95 air and $5 \% \mathrm{CO}_{2}$ at $37{ }^{\circ} \mathrm{C}$. All experiments were performed $24 \mathrm{~h}$ after cells were seeded on 96-well plates at a density of $2.0 \times 10^{4}$ cells/well. Cells were treated with $0.1-100 \mu \mathrm{g} /$ $\mathrm{mL}$ RRs in serum-free media for $4 \mathrm{~h}$. Cell viability was measured using the MTT method [13].

\section{Effects of RRs on rat liver}

Thirty male Sprague-Dawley rats (5 weeks, 120-140 g) were obtained from Orient Bio (Sungnam, Korea). This study was carried out in accordance with the Principles of Laboratory Animal Care and Use Guidelines of Kyung 

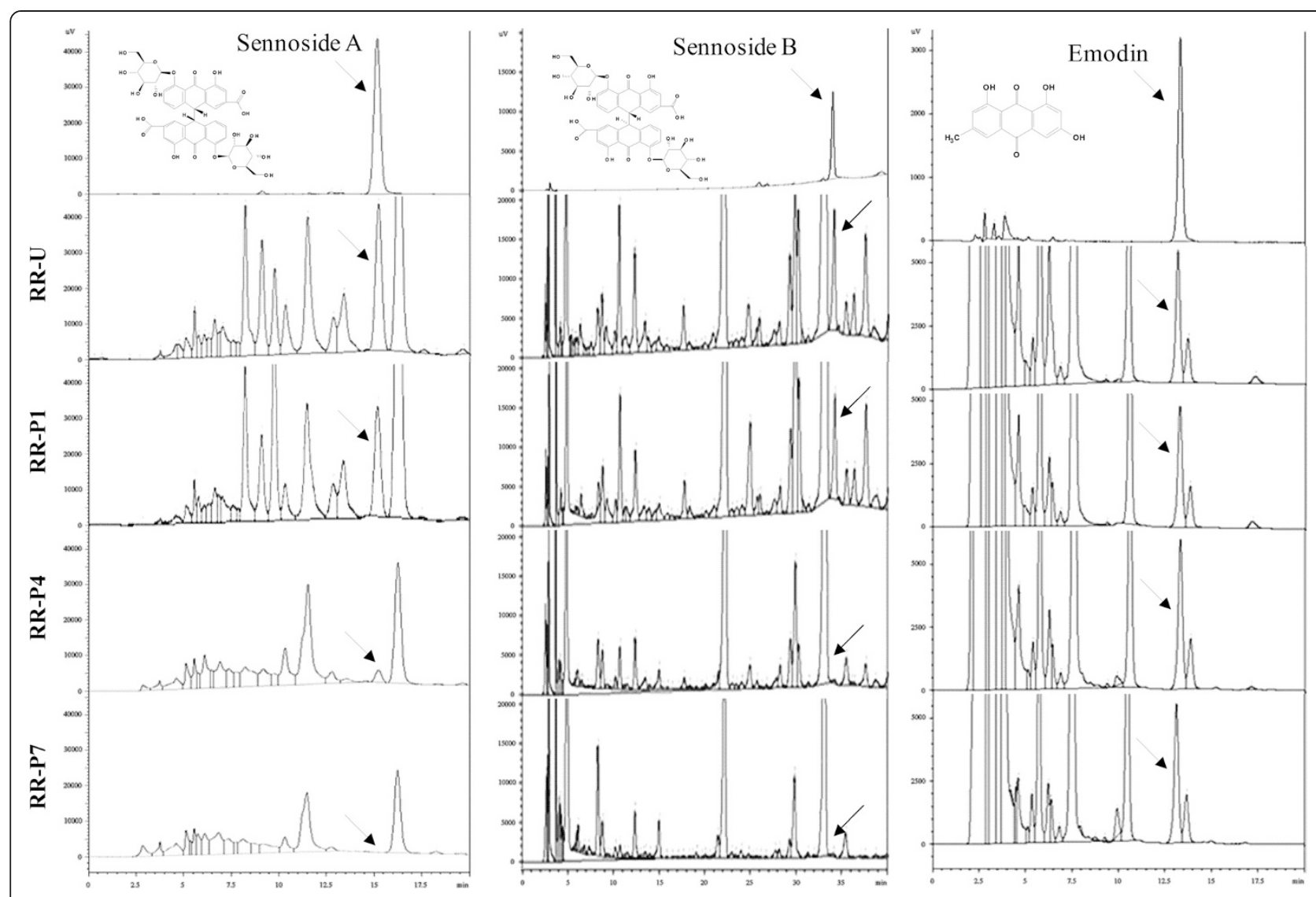

Fig. 1 Sennoside A, sennoside B and emodin constituent changes in unprocessed or processed Rhei Rhizoma

Hee University, and was approved by the Ethics Committee of Kyung Hee University (Seoul, Korea). Animals were housed at an ambient temperature of $23 \pm 1{ }^{\circ} \mathrm{C}$ and at a relative humidity of $60 \pm 10 \%$ under a 12 h light-dark cycle, and they were allowed free access to water and food. Animals were randomized into five groups: (1) control group; (2) RR-U treated group; (3) RR-P1 treated group; (4) RR-P4 treated group; (5) RR-P7 treated group. Vehicle or $3 \mathrm{~g} / \mathrm{kg} /$ day of each sample dissolved in saline was administered orally once a day for 21 days. The treatment dose
$(3 \mathrm{~g} / \mathrm{kg})$ in rats was equivalent to the maximal clinical dose of RR in the Chinese pharmacopoeia $(0.5 \mathrm{~g} / \mathrm{kg})[14,15]$.

On the last day of treatment, blood samples were collected into non heparinized tubes and centrifuged at $3000 \mathrm{rpm}$ for $10 \mathrm{~min}$. The separated serum was analyzed to evaluate the liver enzymes. Alanine aminotransferase (ALT), aspartate aminotransferase (AST), alkaline phosphatase (ALP), total bilirubin (T-BIL), and gamma-glutamyltransferase $(\gamma-\mathrm{GT})$ were entrusted to Chemon (Yongin, Korea) for analyses.
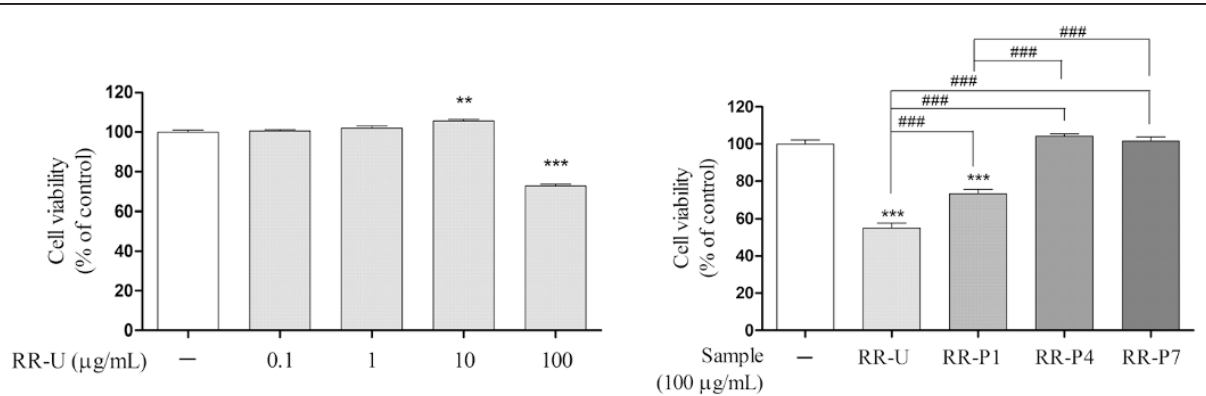

Fig. 2 Effects of unprocessed or processed Rhei Rhizoma on HepG2 cell viability. Cells were treated with RR-U, RR-P1, RR-P4 and RR-P7 at concentration of $100 \mu \mathrm{g} / \mathrm{mL}$ for $4 \mathrm{~h}$. Cell viability was presented as a percentage of the control. ${ }^{* *} p<0.01$ and ${ }^{* * *} p<0.001$ compared with the control group. \#\#\# $p<0.001$ compared with RR-U or RR-P1 treated group 
Table 1 Effect of unprocessed or processed Rhei Rhizoma on rat body and liver weights

\begin{tabular}{llllll}
\hline & Control & RR-U & RR-P1 & RR-P4 & RR-P7 \\
\hline Body weight (g) & $339.53 \pm 6.54$ & $290.48 \pm 7.97^{* * *}$ & $326.83 \pm 9.23^{\# \# \#}$ & $333.77 \pm 12.42^{\# \# \#}$ & $339.18 \pm 10.10^{\# \# \#}$ \\
Liver weight (g) & $14.21 \pm 0.58$ & $14.17 \pm 0.71$ & $13.12 \pm 1.07$ & $13.03 \pm 0.78$ & $15.07 \pm 0.92$ \\
Relative weight (\%) & $4.19 \pm 0.25$ & $4.88 \pm 0.21^{*}$ & $4.01 \pm 0.27^{\# \#}$ & $3.90 \pm 0.24^{\# \#}$ & $4.44 \pm 0.26$ \\
\hline
\end{tabular}

Relative weight: liver weight/body weight $\times 100$. ${ }^{*} p<0.05$ and ${ }^{* * *} p<0.001$ compared with the control group

${ }^{\# \#} p<0.01$ and ${ }^{\# \# \#} p<0.001$ compared with RR-U treated group

Necropsies were performed on all animals. After sacrifice, pieces of all excised tissues were individually placed in neutral buffered formalin for histologic examinations. Tissue specimens were processed into paraffin-embedded $5-\mu \mathrm{m}$ sections and stained with hematoxylin and eosin (H\&E). Sections were fixed in $100 \%$ acetone at $-20{ }^{\circ} \mathrm{C}$ for $15 \mathrm{~min}$. Sections were stained with hematoxylin for $1 \mathrm{~min}$ and destained by dipping in acid ethanol. Then, sections were stained with eosin for $30 \mathrm{~s}$, dehydrated and mounted. The images were photographed at $400 \times$ magnification using an optical light microscope (Olympus Microscope System BX51; Olympus, Tokyo, Japan).

\section{Statistical analysis}

The statistical parameters were produced using GraphPad Prism 5.0 software. Values are presented as mean \pm standard error of the mean (S.E.M.). Results were analyzed by one-way analysis of variance test with Tukey's post hoc option.

\section{Results and discussion}

In this study, we investigated the changes in the sennoside $\mathrm{A}$, sennoside $\mathrm{B}$ and emodin constituents in RRs using RP-HPLC and found that seven-time alcoholsteaming reduced RR hepatotoxicity almost completely in vitro and in vivo.

Regarding changes in constituents after RR-U, RR-P1, RR-P4 and RR-P7, the levels of sennoside A were 13.7, $10.67,1.62$ and $0 \mu \mathrm{g} / \mathrm{mg}$, respectively. The levels of sennoside B after RR-U, RR-P1, RR-P4 and RR-P7 were 8.9, 7.7, 4.6 and $3.8 \mu \mathrm{g} / \mathrm{mg}$, respectively. The levels of emodin after RR-U, RR-P1, RR-P4 and RR-P7 were 5.9, 6.1, 6.3 and $5.8 \mu \mathrm{g} / \mathrm{mg}$, respectively (Fig. 1). Doui et al. compared processing of RR with huangjiu or baijiu [10] and with or without preprocessing with alcohol [11] and speculated that steaming of RR decreased the sennoside contents; moreover, the reduction was greater as steaming time increased because steaming flexes the cells, causing elution of water-soluble compounds. In this study, alcoholsteaming of RR decreased the levels of sennoside A and B in an iteration-dependent manner, while emodin levels were unaffected. Noticeably, the sennoside peaks in RR-P7 almost disappeared.

To evaluate the effect of alcohol-steaming on RR toxicity to HepG2 cells, MTT assay was performed. Significant cell toxicity was observed with $100 \mu \mathrm{g} / \mathrm{mL}$ treatment of RR-U. The toxicity of RR-U was recovered according to the number of alcohol-steaming process (Fig. 2).

To confirm the effects in vivo, $3 \mathrm{~g} / \mathrm{kg}$ of RRs were administered orally to rats for 21 days. The weights of body and liver in rats were measured to evaluate the general and liver-specific toxicity of RRs. The body weight in the RR-U group was significantly lower than that in the control group, while the body weight in the processed RRs groups increased significantly as the process was repeated. The relative weight (liver weight/body weight $\times 100$ ) in the RR-U group was greater than that in the control group, and the weights in the RR-P1 and RR-P4 groups significantly decreased (Table 1). The levels of AST, ALT, ALP, T-BIL and $\gamma$-GT liver enzymes were measured to assess liver function. Serum liver enzymes are the most sensitive markers of hepatic damage because they are located in the cytoplasm and released into the circulation after cellular damage [16]. T-BIL and ALT levels in the RR-U group were higher than those in the control, RR-P4 and RR-P7 groups. The AST levels in the RR-U group were slightly higher, and those in the RR-P7 group were significantly lower, compared with the RR-U group. ALP and $\gamma$-GT levels differed among the groups, albeit not significantly so. Even though the reason for the result that RR-P7 group exhibited a slight increase in $\gamma$-GT compared to those of RRP1 and RR-P4 groups was unclear, the increased level of

Table 2 Effect of unprocessed or processed Rhei Rhizoma on biochemical indices of rats

\begin{tabular}{llllll}
\hline & Control & RR-U & RR-P1 & RR-P4 & RR-P7 \\
\hline T-BIL (mg/dL) & $0.05 \pm 0.01$ & $0.30 \pm 0.07^{* * *}$ & $0.11 \pm 0.01^{\# \#}$ & $0.09 \pm 0.01^{\# \# \#}$ & $0.09 \pm 0.01^{\# \#}$ \\
AST (IU/L) & $94.15 \pm 2.63$ & $100.33 \pm 6.44$ & $86.52 \pm 7.59$ & $88.44 \pm 6.72$ & $81.92 \pm 1.64^{\#}$ \\
ALT (IU/L) & $44.38 \pm 1.68$ & $48.95 \pm 2.01^{*}$ & $46.60 \pm 4.37$ & $42.62 \pm 4.26^{* * \# \#}$ & $38.45 \pm 1.28^{* \# \#}$ \\
ALP (IU/L) & $301.18 \pm 22.60$ & $300.17 \pm 31.66$ & $257.45 \pm 28.04$ & $283.70 \pm 25.88$ & $293.73 \pm 40.03$ \\
$Y-G T(I U / L)$ & $0.02 \pm 0.02$ & $0.14 \pm 0.07$ & $0.02 \pm 0.02$ & $0.02 \pm 0.02$ & $0.09 \pm 0.05$ \\
${ }^{*} p<0.05,{ }^{* *} p<0.01$, and $^{* * * *} p<0.001$ compared with the control group. ${ }^{\# p} p<0.05,{ }^{\# \#} p<0.01$, and ${ }^{\# \# \#<0.001}$ compared with RR-U treated group
\end{tabular}




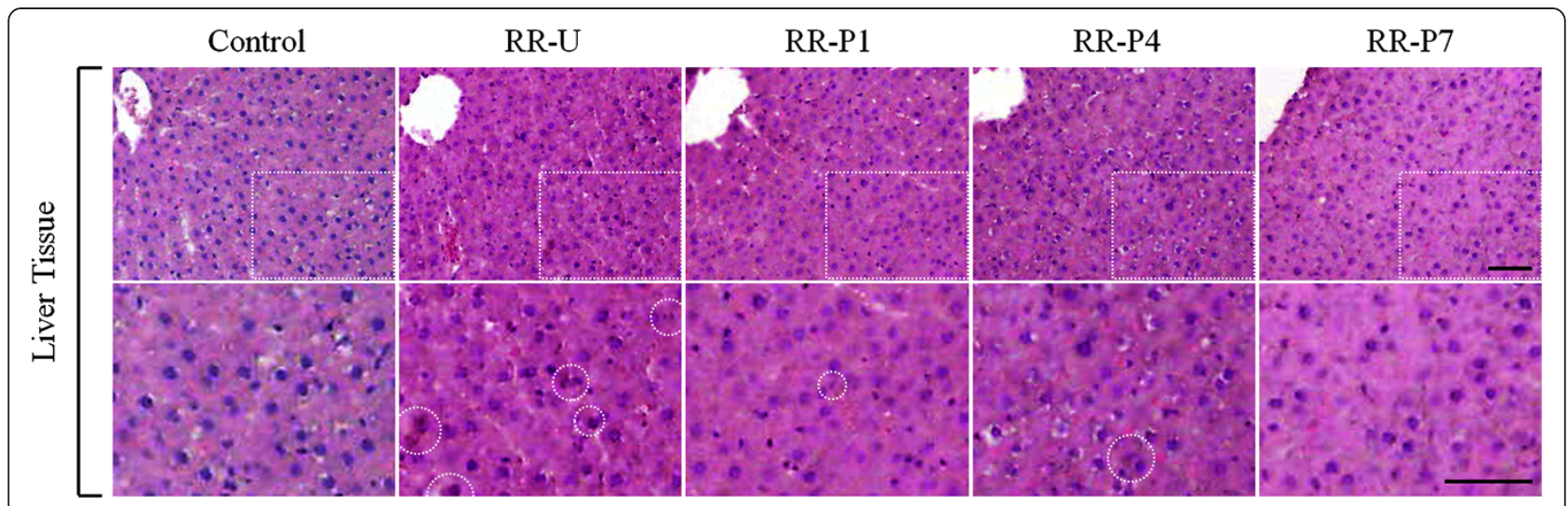

Fig. 3 Liver morphologies of unprocessed or processed Rhei Rhizoma. Typical histopathological features of the rats treated with RR-U, RR-P1, RR-P4 and RR-P7. The yellowish brown bile pigments (white dashed circles). Scale bar $=50 \mu \mathrm{m}$

RR-P7 group was statistically insignificant and still lower than that of RR-U group (Table 2). These findings also suggest that alcohol-steamed RRs affect the hepatobiliary and hematogenous systems. A histopathological study of rats treated with excessive RR-U showed swelling in the liver [4]. In this study, morphological changes in the liver were examined using H\&E staining to evaluate the effect of RRs on liver toxicity. Stained liver tissues showed swelling and yellowish brown bile pigment indicating cholestasis in the rats treated with RR-U, while this phenomenon improved in the groups treated with processed RRs as the process was repeated (Fig. 3).

Furthermore, to investigate the efficacy of RRs after processing, we performed supplementary experiments to compare the anti-oxidative and neuroprotective activities of RRs. RRs exhibited strong radical scavenging activities, even anti-oxidative activities of RR-P7 were higher than those of the water extract of Scutellariae Radix (SBE), a positive control, which is well-known anti-oxidant and neuroprotectant [17, 18] (Additional file 1A). Also, we compared the protective effects of RRs against $\mathrm{H}_{2} \mathrm{O}_{2}$-induced neurotoxicity in PC12 cells. Decreased cell viability induced by $\mathrm{H}_{2} \mathrm{O}_{2}$ was inhibited by RRs pre-treatment, showing a better effect than SBE (Additional file 1B). These results suggest that the efficacy of RRs after processing may be still potent.

In this study, alcohol-steaming of RRs reduced their hepatotoxicity, which was normalized in vitro and in vivo after RR-P7 treatment due to the decreased the levels of sennoside A and B and maintained emodin levels. Sennosides have been reported to be related to hepatotoxicity [19]. Emodin, an anthraquinone derivative of RR, has anti-oxidant, anti-inflammatory and hepatoprotective effects [20-23]. Thus, the changes in chemical constituents suggest that the seven-time alcohol-steaming process reduced the hepatotoxicity of RR-U.

\section{Conclusions}

The decreased hepatotoxicity of RR was related to the number of alcohol-steaming cycles; the seven-time alcohol-steaming process almost completely removed its hepatotoxicity. In this study, using modern pharmacological, toxicological and chemical analyses, we confirmed that RR required alcohol-steamed seven times and provided a scientific evidence for conducting systematic investigations of ancient documents.

\section{Additional file}

Additional file 1: Radical scavenging activities and neuroprotective effects of unprocessed or processed Rhei Rhizoma. (PDF $96 \mathrm{~kb}$ )

\section{Competing interests}

The authors declared that there is no conflict of interests regarding the publication of this article.

\section{Authors' contributions}

Myung Sook Oh advised the concept, designed the study, critically revised the manuscript, and is responsible for the study. Hyein Oh, Tong Ho Kang, and Yeomoon Sim performed the experiments and analyzed the data. Namkwon Kim, Pil Sung Gu, and Jin Gyu Choi performed the supplementary experiments and analyzed the data. Hyo Geun Kim, Yeomoon Sim and Hyein Oh conceptualized the research question, wrote the first draft of the manuscript. Tong Ho Kang and Dal-Seok Oh critically rewrote the manuscript. All the authors read and approved the final version of the manuscript.

\section{Acknowledgements}

This study was supported by the Bio-Synergy Research Project (NRF-2012M3A9C4048795) of the Ministry of Science, ICT, and Future Planning through the National Research Foundation.

\section{Author details}

'Department of Life and Nanopharmaceutical Science, Graduate School, Kyung Hee University, 26 Kyungheedae-ro, Dongdaemun-gu, Seoul 02447 Republic of Korea. ${ }^{2}$ Division for Medical Research, Korea Institute of Oriental Medicine, Daejeon, Republic of Korea. ${ }^{3}$ Department of Oriental

Pharmaceutical Science, College of Pharmacy and Kyung Hee East-west Pharmaceutical Research Institute, Kyung Hee University, 26 Kyungheedae-ro, Dongdaemun-gu, Seoul 02447, Republic of Korea. ${ }^{4}$ Department of Oriental 
Medicinal Materials \& Processing, College of Life Sciences, Kyung Hee University, Gyeonggi-do, Republic of Korea.

Received: 25 May 2015 Accepted: 19 October 2015

Published online: 27 October 2015

\section{References}

1. Xiao P, He L, Wang L. Ethnopharmacologic study of Chinese rhubarb. J Ethnopharmacol. 1984;10(3):275-93.

2. Ye M, Han J, Chen H, Zheng J, Guo D. Analysis of phenolic compounds in rhubarbs using liquid chromatography coupled with electrospray ionization mass spectrometry. J Am Soc Mass Spectrom. 2007;18(1):82-91.

3. Clementi EM, Misiti F. Potential health benefits of rhubarb. In: Watson RR, Preedy VR, editors. Bioactive foods in promoting health: fruits and vegetables. Waltham, MA: Academic; 2010. p. 407-23.

4. Jin K, Tian Y, Xin J, Inoue M, Setsu K, Rui K, et al. Hepatotoxicity induced by excessive intake of rhubarb. J Hard Tissue Biology. 2006;15(1):16-9.

5. Yan M, Zhang LY, Sun LX, Jiang ZZ, Xiao XH. Nephrotoxicity study of total rhubarb anthraquinones on Sprague Dawley rats using DNA microarrays. J Ethnopharmacol. 2006;107(2):308-11.

6. Zhao Z, Liang Z, Chan K, Lu G, Lee ELM, Chen H, et al. A unique issue in the standardization of Chinese materia medica: processing. Planta Med. 2010;76(17):1975-86.

7. Wang J, Ma Y, Jin C, Zhang P, Xiao X, Zhao Y, et al. Study toxicity-attenuating effect and dose-toxicity relationship of rhubarb by processing based on correspondence analysis. Zhongguo Zhong Yao Za Zhi. 2009;34(19):2498-502.

8. Wang G, Hou Z, Peng Y, Wang Y, Sun X, Sun YA. Adaptive kernel independent component analysis and UV spectrometry applied to characterize the procedure for processing prepared rhubarb roots. Analyst. 2011;136:4552-7.

9. Seo BI, Kwon DY, Choi HY, Lee JH, Oh MS, Bu YM. Medicinal Herbology. 1st ed. Younglim-Sa: Seoul; 2012.

10. Doui M, Kakiuchi N, Mikage M. Studies on rhubarb processed with liquor-The reason why rhubarb is processed with huangjiu: the liquor contains a small amount of alcohol. J Trad Med. 2009;26(4):160-8.

11. Doui M, Kakiuchi N, Mikage M. Chemical differences between steamed rhubarbs with or without pre-processing with liquor. J Trad Med. 2010;27(3):109-14.

12. Heo J. Translated Dongeuibogam. 1st ed. Bubinmunwha: Seoul; 1999.

13. Park G, Kim HG, Sim Y, Sung SH, Oh MS. Sauchinone, a lignan from Saururus chinensis, protects human skin keratinocytes against ultraviolet B-induced photoaging by regulating the oxidative defense system. Biol Pharm Bull. 2012;36(7):1134-9.

14. Kuang L, Zhang K, C.P. Commission. Pharmacopoeia of the People's Republic of China 2005. Beijing: People's Medical Publishing House; 2005.

15. Shin JW, Seol IC, Son CG. Interpretation of animal dose and human equivalent dose for drug development. J Korean Med. 2010;31(3):1-7.

16. Pradeep K, Mohan CVR, Gobianand K, Karthikeyan S. Effect of Cassia fistula Linn. leaf extract on diethylnitrosamine induced hepatic injury in rats. Chem-Biol Interact. 2007;167(1):12-8

17. Gao Z, Huang K, Yang X, Xu H. Free radical scavenging and antioxidant activities of flavonoids extracted from the radix of Scutellaria baicalensis Georgi. Biochim Biophys Acta. 1999;1472(3):643-50.

18. Shang YZ, Qin BW, Cheng JJ, Miao H. Prevention of oxidative injury by flavonoids from stems and leaves of Scutellaria baicalensis Georgi in PC12 cells. Phytother Res. 2006;20(1):53-7

19. Vanderperren B, Rizzo M, Angenot L, Haufroid V, Jadoul M, Hantson P. Acute liver failure with renal impairment related to the abuse of senna anthraquinone glycosides. Ann Pharmacother. 2005;39(7-8):1353-7.

20. Yen GC, Duh PD, Chuang DY. Antioxidant activity of anthraquinones and anthrone. Food Chem. 2000;70(4):437-41.

21. Chang CH, Lin CC, Yang JJ, Namba T, Hattori M. Anti-inflammatory effects of emodin from ventilago leiocarpa. Am J Chin Med. 1996;24(2):139-42.

22. Dang SS, Zhang $X$, Jia XL, Cheng YA, Song $P$, Liu EQ, et al. Protective effects of emodin and astragalus polysaccharides on chronic hepatic injury in rats. Chin Med J. 2008:121(11):1010-4.

23. Zhao Y, Wang J, Zhou G, Shan L, Xiao X. Investigations of free anthraquinones from rhubarb against a-naphthylisothiocyanate-induced cholestatic liver injury in rats. Basic Clin Pharmacol Toxicol. 2009;104(6):463-9.

\section{Submit your next manuscript to BioMed Central and take full advantage of:}

- Convenient online submission

- Thorough peer review

- No space constraints or color figure charges

- Immediate publication on acceptance

- Inclusion in PubMed, CAS, Scopus and Google Scholar

- Research which is freely available for redistribution

Submit your manuscript at www.biomedcentral.com/submit 